\title{
Imaging of Dentate Nucleus Pathologies: A Case Series
}

\author{
Usha Chowdary M.K. ${ }^{1} \quad$ Naveen Prasad S.V. ${ }^{1} \quad$ Satish Gudla ${ }^{1}$ Vengamma Bhuma ${ }^{1}$ \\ 1 Department of Neurology, Sri Venkateswara Institute of Medical \\ Sciences, Tirupati, Andhra Pradesh, India \\ J Neurosci Rural Pract 2022;13:146-150. \\ Address for correspondence Usha Chowdary M.K., MD, DM, \\ Department of Neurology, Sri Venkateswara Institute of Medical \\ Sciences, Tirupati, Andhra Pradesh 517507, India \\ (e-mail: usha.madineni123@gmail.com).
}
Abstract
Keywords
- computed tomography
- dentate nucleus
- magnetic resonance imaging

The dentate nucleus is the largest cerebellar nucleus, and it controls cognition and voluntary movement. It is found in each cerebellar hemisphere medially and posterolateral to the lateral ventricle. Pathologies of the dentate nucleus can be detected using computed tomography and magnetic resonance imaging of the brain. Here, we present a case series of seven different dentate nucleus diseases and their neuroimaging findings recovered from archives of our institution.

\section{Introduction}

The largest and the most lateral of the four deep cerebellar nuclei is the dentate nucleus, located next to the vermis and posterolateral to the fourth ventricle. ${ }^{1}$ The dentate nucleus is a component of the cerebro-cerebellar circuitry, which relays information to the cortical and subcortical structures. ${ }^{1}$ It has domains for both motor and non-motor functions and is involved in motor movement planning and execution, cognition, and visuospatial sensory processing. ${ }^{1}$ The dentate nucleus can be affected by a variety of pathologies. Its involvement in imaging can be isolated or associated with lesions in other supra and/or infratentorial structures; thus, imaging aids in the formulation of differential diagnoses. Furthermore, lesions in the dentate nucleus may determine hypertrophic olivary degeneration and other transaxonal degenerations. ${ }^{2}$ This article reviews several possible causes of dentate nucleus lesions based on the neuroimaging studies recovered from the archives of our institution.

\section{Cases' Presentations}

\section{Case 1}

\section{Clinical Picture}

A 54-year-old man was on regular peritoneal dialysis for endstage renal disease (ESRD). He was diagnosed to have pulmonary tuberculosis (PTB) and was started on a four-drug antitubercular medication regimen that included isoniazid, rifampicin, pyrazinamide, and ethambutol. After 2 weeks, he developed symptoms of confusion, dysarthria, and gait imbalance as well as signs of bilateral horizontal gaze-evoked nystagmus, dysmetria and past-pointing in all limbs, and ataxic gait.

\section{Imaging Findings}

In magnetic resonance imaging (MRI) of the brain, T2weighted imaging (WI) revealed symmetric hyperintensity involving the cerebellum's bilateral dentate nuclei (-Fig. 1A). There was no diffusion restriction on diffusion- published online January 5, 2022
DOI https://doi.org/ 10.1055/s-0041-1740611. ISSN 0976-3147.

\footnotetext{
(C) 2022. Association for Helping Neurosurgical Sick People. All rights reserved.

This is an open access article published by Thieme under the terms of the Creative Commons Attribution-NonDerivative-NonCommercial-License, permitting copying and reproduction so long as the original work is given appropriate credit. Contents may not be used for commercial purposes, or adapted, remixed, transformed or built upon. (https://creativecommons.org/ licenses/by-nc-nd/4.0/)

Thieme Medical and Scientific Publishers Pvt. Ltd., A-12, 2nd Floor, Sector 2, Noida-201301 UP, India
} 


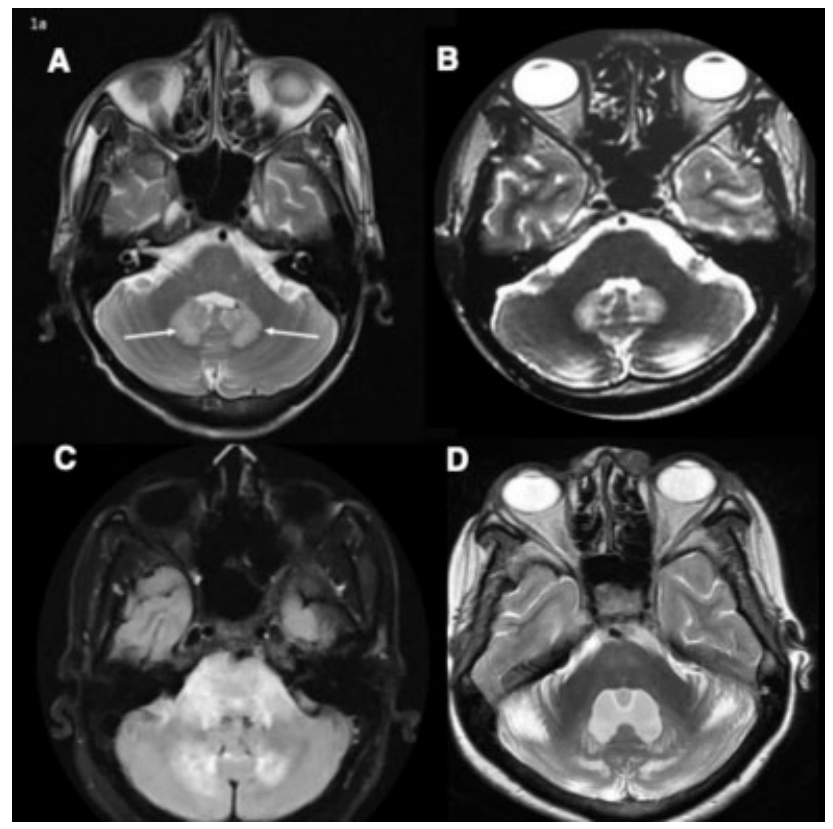

Fig. 1 (A) MRI brain T2 weighted image (WI) showing symmetric hyperintensities in bilateral dentate nuclei in isoniazid toxicity. (B) MRI brain T2WI showing symmetric hyperintensities in bilateral dentate nuclei in ornidazole toxicity. (C) MRI brain FLAIR image showing hyperintensities in bilateral dentate nuclei, middle cerebellar peduncle, and pons in acute disseminated encephalomyelitis. (D) MRI brain T2WI showing symmetric hyperintensities in bilateral dentate nuclei and cerebellar hemispheres in cerebrotendinous xanthomatosis. Bilateral cerebellar hemispheres have prominent folia suggestive of cerebellar atrophy.

weighted imaging (DWI)/apparent diffusion coefficient (ADC) mapping and there was no blooming on susceptibility weighted imaging (SWI) (image not shown). The rest of the brain parenchyma, including the midbrain and thalamus, was normal (-Table 1). After a provisional diagnosis of isoniazid (INH) toxicity, the drug was discontinued.

\section{Discussion}

After 4 weeks, the patient's symptoms typically resolved. INH toxicity is caused by a lack of energy and a deficiency of vitamin B complex. Pyridoxal 5-phosphate synthesis is reduced, which is required for gamma-aminobutyric acid (GABA) neurotransmission. GABA is an inhibitory neurotransmitter that induces cerebellar symptoms when it is in the central nervous system. ${ }^{3}$

\section{Diagnosis}

Isoniazid-induced encephalopathy and cerebellitis in a patient with ESRD and PTB manifested as symmetrical dentate nuclei hyperintensities on both sides, which resolved once the drug was stopped.

\section{Case 2}

\section{Clinical Picture}

A 28-year-old man underwent surgical hemorrhoidectomy. Since then, he has been taking ornidazole on a daily basis.
After 3 months, he experienced new-onset symptoms of confusion, vertigo, and gait imbalance. He did not have seizures or peripheral neuropathy. His vitals and systemic examination were normal. The Glasgow Coma Scale (GCS) score was $13 / 15$ (eye 3, motor 6 , and verbal 4). Neurological examination revealed dysarthria, bilateral horizontal gazeevoked nystagmus, impaired finger-nose test in all limbs, and ataxic gait.

\section{Imaging Findings}

MRI brain T2 WI showed symmetric hyperintensity involving bilateral dentate nuclei (-Fig. 1B). There was no diffusion restriction on DWI and ADC mapping and there was no blooming on SWI imaging (image not shown). The rest of the brain parenchyma including the supratentorial white matter, corpus callosum, and midbrain, was normal. (-Table 1). Ornidazole was withdrawn, and the patient showed typical clinical improvement and resolution of lesions on MRI.

\section{Discussion}

Ornidazole toxicity is primarily caused by mitochondrial dysfunction, free-radical damage, and impairment of GABAergic neurotransmission in the cerebellum and brainstem, which results in cytotoxic edema and localized axonal swelling. ${ }^{4}$

\section{Diagnosis}

Ornidazole toxicity manifested itself as encephalopathy, cerebellitis, and bilateral dentate nucleus hyperintensities, all of which disappeared once the drug was stopped.

\section{Case 3}

\section{Clinical Picture}

A 39-year-old woman had a short-duration fever 15 days ago and recovered spontaneously. She had not received any vaccines and had not experienced any infections requiring treatment recently. She had abrupt onset drowsiness and was unable to stand or walk on her own. Her vitals and systemic examination findings were normal. The GCS score was $13 / 15$ (eye 3, motor 5 , and verbal 4 ). On neurological evaluation, she was disoriented to time, place, and person. She also had dysarthria and horizontal nystagmus and was uncooperative for the rest of the examination.

\section{Imaging Findings}

MRI brain fluid-attenuated inversion recovery (FLAIR) sequence revealed hyperintensity in the bilateral dentate nuclei, cerebellum, middle cerebellar peduncles, pons (-Fig. 1C), and supratentorial white matter (bilateral corona radiata, centrum semiovale, sub-cortical white matter of bilateral frontal, and temporal lobes). However, there was no diffusion restriction, blooming, or post-contrast enhancement in the corresponding areas. Bilateral thalami and the rest of the brain were normal (images not shown) (-Table 1). 


\begin{tabular}{|c|c|c|c|c|c|c|c|}
\hline 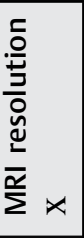 & $\begin{array}{l}\text { 㒸 } \\
\vec{\sigma}\end{array}$ & $\begin{array}{l}\stackrel{y}{3} \\
\vec{\sigma}\end{array}$ & 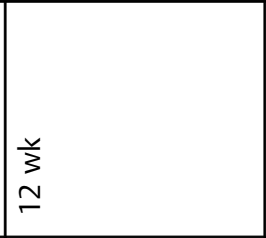 & 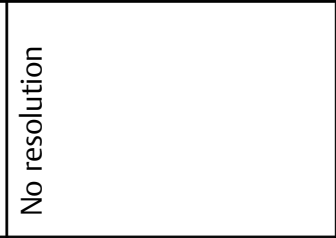 & 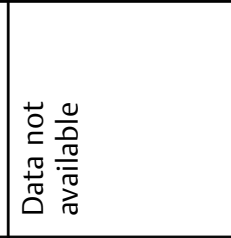 & 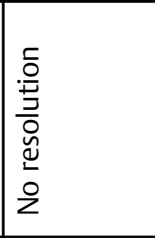 & $\begin{array}{l}\stackrel{y}{3} \\
\vec{\sigma}\end{array}$ \\
\hline 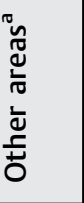 & 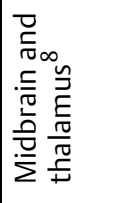 & 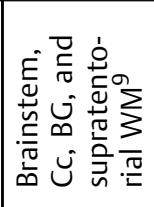 & 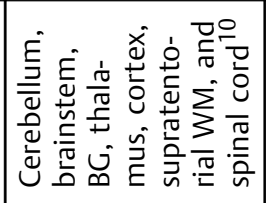 & 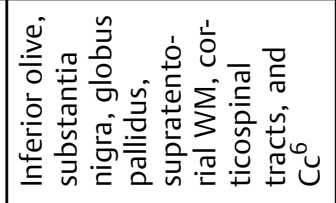 & 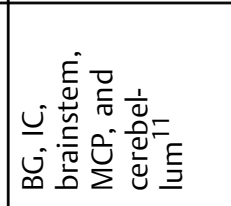 & 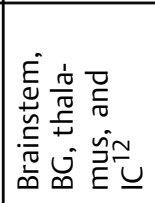 & 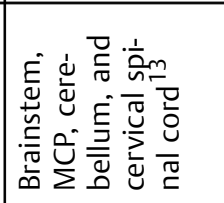 \\
\hline$\sum_{n}$ & 1 & 1 & 1 & 1 & 1 & \multirow{2}{*}{ 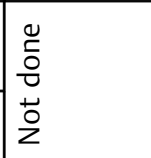 } & $>$ \\
\hline 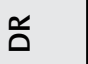 & 1 & 1 & 1 & 1 & 1 & & 1 \\
\hline$\sum_{\xi}$ & 1 & 1 & 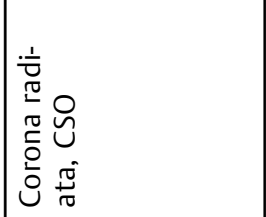 & 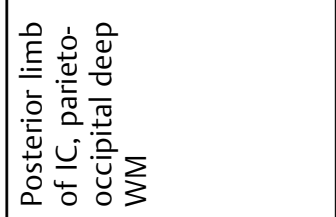 & 1 & $>$ & 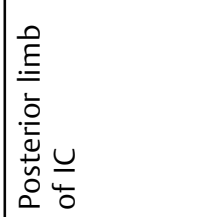 \\
\hline טיه & 1 & 1 & 1 & 1 & 1 & $>$ & 1 \\
\hline 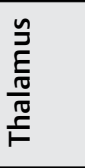 & 1 & 1 & 1 & 1 & 1 & $>$ & 1 \\
\hline$\frac{.5}{\frac{\pi}{0}}$ & 1 & 1 & 1 & 1 & 1 & 1 & $>$ \\
\hline 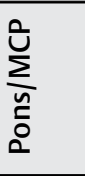 & 1 & 1 & $\sum_{\substack{u \\
\vdots \\
\vdots \\
\vdots}}$ & 1 & $\frac{\bar{U}}{\Sigma}$ & 1 & $\sum_{\substack{u \\
\tilde{L} \\
\vdots \\
\Sigma}}$ \\
\hline$\frac{\frac{\pi}{\bar{z}}}{\overline{\bar{\sigma}}}$ & 1 & 1 & 1 & 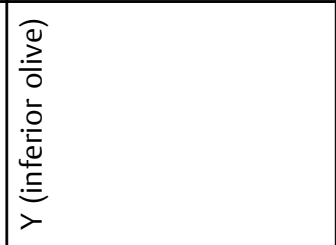 & 1 & 1 & $>$ \\
\hline 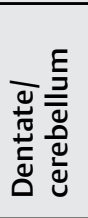 & $>$ & $>$ & 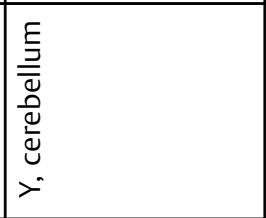 & 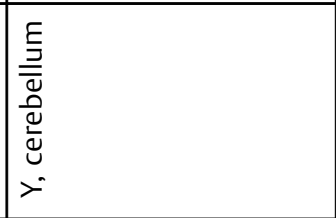 & $>$ & 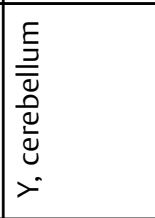 & 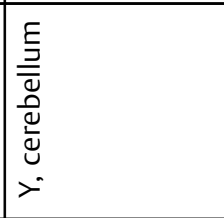 \\
\hline 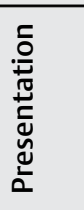 & 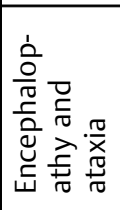 & 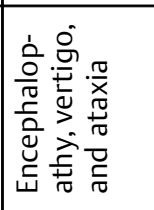 & 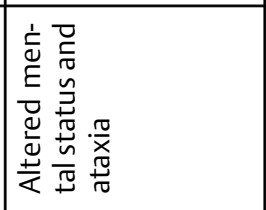 & 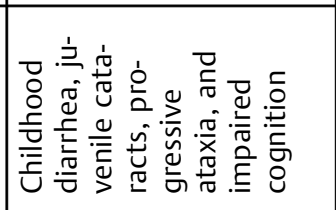 & 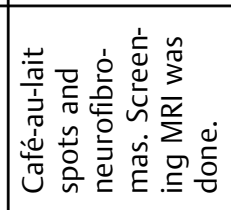 & 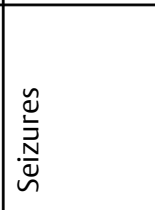 & 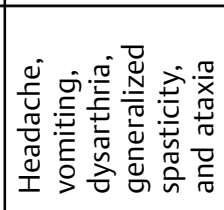 \\
\hline $\begin{array}{l}\frac{n}{\tilde{n}} \\
\frac{0}{5} \\
\frac{\pi}{0}\end{array}$ & 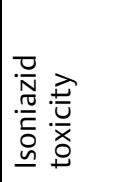 & 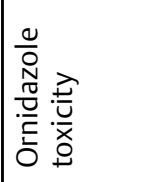 & 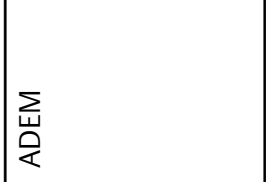 & $\stackrel{x}{e}$ & 立 & 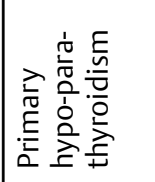 & 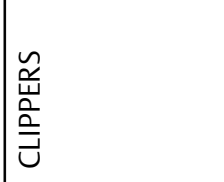 \\
\hline $\begin{array}{l}\tilde{n} \\
\tilde{U}\end{array}$ & - & $\sim$ & $m$ & $\nabla$ & in & 0 & $\wedge$ \\
\hline
\end{tabular}




\section{Discussion}

Acute disseminated encephalomyelitis (ADEM) was the preliminary diagnosis. She was treated with a $1 \mathrm{~g} /$ day dose of methylprednisolone for 5 days and was switched to oral prednisolone, $40 \mathrm{mg} /$ day, which was slowly tapered over a period of 3 months and discontinued. After 3 months, the patient's symptoms improved significantly, and the lesions resolved on MRI. Okumura et al reported a case study of two patients with ADEM and severe brain involvement, including dentate nuclei. ${ }^{5}$

\section{Diagnosis}

Diffuse brain including bilateral dentate nuclei hyperintensities in a patient with ADEM.

\section{Case 4}

\section{Clinical Picture}

A 47-year-old woman experienced symptoms of progressive unsteadiness and abnormal behavior over the past 9 years. As a toddler, she had suffered from severe diarrhea and later developed juvenile cataracts. During systemic and neurological evaluation, she was found to have multiple xanthomas, impaired cognition, horizontal gaze-evoked nystagmus, dysmetria and past-pointing in all four limbs, and ataxic gait. Her younger sister is afflicted with the same condition.

\section{Imaging Findings}

An MRI brain T2 sequence revealed symmetric hyperintensity in the bilateral dentate nucleus ( - Fig. 1D), inferior olive, cerebellar hemisphere, posterior limb of internal capsule, and parieto-occipital deep white matter (images not shown). Nonetheless, there was no diffusion restriction, blooming, or contrast enhancement in these hyperintensities. The rest of the brain including globus pallidus and substantia nigra was normal (-Table 1). MRI orbit T1/T2 WI showed the presence of a hypointense lesion in the lower eyelid, which was suggestive of xanthoma.

\section{Discussion}

The presence of chronic diarrhea, juvenile cataracts, tendon xanthomas, progressive cerebellar ataxia, and cognitive decline suggested cerebrotendinous xanthomatosis (CTX). Pudhiavan et al. reported similar clinical and imaging findings in CTX in his case report. ${ }^{6}$

\section{Diagnosis}

\section{CTX}

\section{Case 5}

\section{Clinical Picture}

A 5-year-old girl had multiple café-au-lait spots and a few neurofibromas. She was diagnosed with neurofibromatosis type 1 (NF1) based on its diagnostic criteria. Her neurological evaluation was unremarkable.

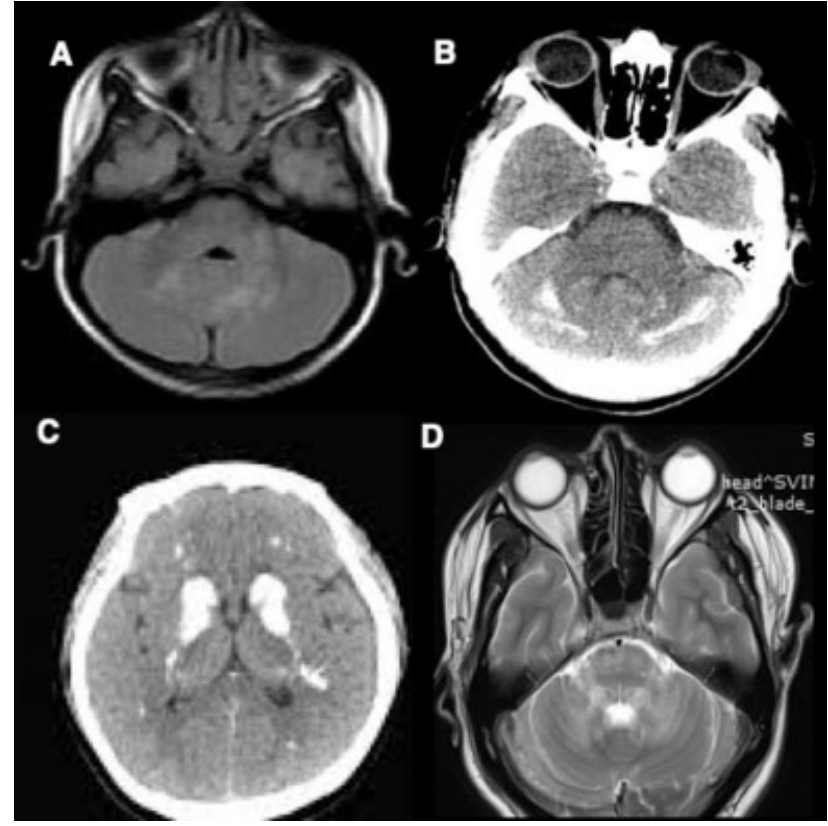

Fig. 2 (A) MRI brain T2 weighted image (WI) showing symmetric hyperintensities in bilateral dentate nuclei and left middle cerebellar peduncle in Neurofibromatosis type 1. (B) CT brain showing hyperdensities in bilateral dentate nuclei and cerebellar hemispheres in primary hypoparathyroidism. (C) CT brain showing hyperdensities in bilateral basal ganglia and supratentorial white matter in primary hypoparathyroidism. (D) MRI brain T2WI showing symmetric hyperintensities in bilateral dentate nuclei, middle cerebellar peduncle, and pons in chronic lymphocytic inflammation with pontine perivascular enhancement responsive to steroids.

\section{Imaging Findings}

MRI brain T2WI/FLAIR revealed hyperintensities in the bilateral dentate nuclei and left middle cerebellar peduncle (-Fig. 2A) without diffusion restriction. There was no blooming on SWI and there was no post-contrast enhancement. The rest of the brain was normal (-Table 1). There were no other NF1 features, either clinically or on brain/whole spine imaging

\section{Discussion}

Takashi Itoh described a range of imaging abnormalities in NF1. The lesions primarily affect the cerebellar hemisphere and the dentate nucleus in the first decade and never after the third decade. ${ }^{7}$

\section{Diagnosis}

Bilateral dentate nucleus hyperintensities were identified on screening brain/spine MRI in a patient with NF1.

\section{Case 6}

\section{Clinical Picture}

A 36-year-old woman receiving regular treatment for primary hypoparathyroidism experienced hypocalcemia symptoms as well as multiple generalized tonic-clonic seizures. Trousseau's and Chvostek's signs were both present. The neurological exam was unremarkable. 


\section{Imaging Findings}

The 36-year-old woman's CT brain showed hyperdense signal in the bilateral dentate nuclei, cerebellar hemispheres ( - Fig. 2B), basal ganglia, and supratentorial white matter (-Fig. 2C). These imaging findings were suggestive of calcification (-Table $\mathbf{1})$.

\section{Discussion}

Primary hypoparathyroidism is a well-known cause of metabolic intracranial brain calcifications.

\section{Diagnosis}

A patient with primary hypoparathyroidism and seizure disorder had pathological intracranial calcification involving the diffuse brain parenchyma and including the bilateral dentate nuclei.

\section{Case 7}

\section{Clinical Picture}

A 23-year-old man had suffered from severe headache and vomiting for 4 weeks. He also experienced a slight unsteadiness while walking. Neurological evaluation revealed bilateral gaze-evoked nystagmus, pyramidal signs in the form of generalized spasticity, hyperreflexia, and bilateral extensor plantar response and cerebellar signs in the form of dysmetria and past-pointing involving all the limbs and gait ataxia.

\section{Imaging Findings}

On MRI brain plain and post-contrast imaging, T2WI/FLAIR showed symmetric hyperintensity in the dentate nucleus, middle cerebellar peduncle, pons (-Fig. 2D), and midbrain without any diffusion restriction on DWI/ADC mapping. Furthermore, the pons and midbrain showed diffuse punctate nodular enhancement. On SWI, there were multiple punctate areas of blooming in the pons (images not shown) (-Table $\mathbf{1}$ ).

\section{Discussion}

The patient responded to intravenous methylprednisolone $1 \mathrm{~g} /$ day for 5 days. A follow-up MRI performed after 4 weeks showed near-complete resolution. He was diagnosed with chronic lymphocytic inflammation with pontine perivascular enhancement sensitive to steroids (CLIPPERS) based on imaging characteristics and steroid responsiveness.

\section{Diagnosis \\ CLIPPERS.}

\section{Funding \\ None}

Conflict of Interest

None declared.

\section{References}

1 Bond KM, Brinjikji W, Eckel LJ, Kallmes DF, McDonald RJ, Carr CM. Dentate update: imaging features of entities that affect the dentate nucleus. AJNR Am J Neuroradiol 2017;38(08): 1467-1474

2 Raeder MTL, Reis EP, Campos BM, Zamilute IAG, França Júnior MC, Reis F. Transaxonal degenerations of cerebellar connections: the value of anatomical knowledge. Arq Neuropsiquiatr 2020;78(05): 301-306

3 Wang HY, Chien CC, Chen YM, Huang CC. Encephalopathy caused by isoniazid in a patient with end stage renal disease with extrapulmonary tuberculosis. Ren Fail 2003;25(01):135-138

4 Mittal GK, Sureshbabu S, Peter S, Khanna L. Dentate hyperintensities-the imaging signature of metronidazole induced encephalopathy. J Neurosci Rural Pract 2017;8(02):272-273

5 Okumura A, Hayakawa M, Watanabe K, Kito M, Negoro T, Kawamura M. [Two cases of acute disseminated encephalomyelitis with lesions in the thalamus or basal ganglia on MRI]. No To Hattatsu 1992;24(03):278-282

6 Pudhiavan A, Agrawal A, Chaudhari S, Shukla A. Cerebrotendinous xanthomatosis-the spectrum of imaging findings. J Radiol Case Rep 2013;7(04):1-9

7 Itoh T, Magnaldi S, White RM, et al. Neurofibromatosis type 1: the evolution of deep gray and white matter MR abnormalities. AJNR Am J Neuroradiol 1994;15(08):1513-1519

8 Hasiloglu ZI, Albayram S, Asik M, et al. MRI findings of isoniazidinduced central nervous system toxicity in a child. Clin Radiol 2012;67(09):932-935

9 Patel L, Batchala P, Almardawi R, Morales R, Raghavan P. Acute metronidazole-induced neurotoxicity: an update on MRI findings. Clin Radiol 2020;75(03):202-208

10 Acute Disseminated Encephalomyelitis - StatPearls - NCBI Bookshelf [Internet]. Accessed October 23, 2021 at: https://www.ncbi. nlm.nih.gov/books/NBK430934/

11 Ferner RE, Chaudhuri R, Bingham J, Cox T, Hughes RA. MRI in neurofibromatosis 1 . The nature and evolution of increased intensity $\mathrm{T} 2$ weighted lesions and their relationship to intellectual impairment. J Neurol Neurosurg Psychiatry 1993;56(05): 492-495

12 Rizvi I, Ansari NA, Beg M, Shamim MD. Widespread intracranial calcification, seizures and extrapyramidal manifestations in a case of hypoparathyroidism. N Am J Med Sci 2012;4(08):369-372

13 Tobin WO, Guo Y, Krecke KN, et al. Diagnostic criteria for chronic lymphocytic inflammation with pontine perivascular enhancement responsive to steroids (CLIPPERS). Brain 2017;140(09): $2415-2425$ 